\title{
Adrenal incidentaloma - follow-up results from a Swedish prospective study
}

\author{
Birgitta Bülow, Svante Jansson ${ }^{1}$, Claes Juhlin ${ }^{2}$, Lars Steen ${ }^{3}$, Marja Thorén ${ }^{4}$, Hans Wahrenberg ${ }^{5}$, \\ Stig Valdemarsson ${ }^{6}$, Bo Wängberg ${ }^{1}$ and Bo Ahrén ${ }^{6}$ on behalf of the Swedish Research Council Study \\ Group of Endocrine Abdominal Tumours \\ Department of Medicine, Ryhov Hospital, 55185 Jönköping, Sweden, ${ }^{1}$ Department of Surgery, Sahlgrenska University Hospital, Gothenburg, Sweden, \\ ${ }^{2}$ Department of Surgery, University Hospital, Linköping, Sweden, ${ }^{3}$ Department of Medicine, Eskilstuna Hospital, Sweden, ${ }^{4}$ Department of Endocrinology, \\ Metabolism and Diabetes, Karolinska University Hospital, Solna, Sweden, ${ }^{5}$ Department of Endocrinology, Metabolism and Diabetology, Karolinska \\ University Hospital, Huddinge, Sweden and ${ }^{6}$ Department of Endocrinology and Diabetology, University Hospital, Lund, Sweden
}

(Correspondence should be addressed to B Bülow; Email: b.bulow@telia.com)

\begin{abstract}
Objectives: To examine the risk of developing adrenal carcinomas and clinically overt hypersecreting tumours during short-term follow-up in patients with adrenal incidentalomas.

Design: 229 (98 males and 131 females) patients with adrenal incidentalomas were investigated in a prospective follow-up study (median time 25 months; range 3-108 months). The patients were registered between January 1996 and July 2001 and followed until December 2004. Twenty-seven Swedish hospitals contributed with follow-up results.

Methods: Diagnostic procedures were undertaken according to a protocol including reinvestigation with computed tomography scans after 3-6 months, 15-18 months and 27-30 months, as well as hormonal evaluation at baseline and after 27-30 months of follow-up. Operation was recommended when the incidentaloma size increased or if there was a suspicion of a hypersecreting tumour.

Results: The median age at diagnosis of the 229 patients included in the follow-up study was 64 years (range 28-84 years) and the median size of the adrenal incidentalomas when discovered was $2.5 \mathrm{~cm}$ (range $1-8 \mathrm{~cm}$ ). During the follow-up period, an increase in incidentaloma size of $\geq 0.5 \mathrm{~cm}$ was reported in $17(7.4 \%)$ and of $\geq 1.0 \mathrm{~cm}$ was reported in $12(5.2 \%)$ of the 229 patients. A decrease in size was seen in 12 patients (5.2\%). A hypersecreting tumour was found in $2 \%$ of the hormonally investigated patients: Cushing's syndrome $(n=2)$ and phaeochromocytoma $(n=1)$. Eleven patients underwent adrenalectomy, but no cases of primary adrenal malignancy were observed.

Conclusions: Patients with adrenal incidentaloma had a low risk of developing malignancy or hormonal hypersecretion during a short-term follow-up period.

European Journal of Endocrinology 154 419-423
\end{abstract}

\section{Introduction}

Adrenal incidentalomas are defined as accidentally discovered clinically silent adrenal lesions present on abdominal imaging that has been performed for reasons other than suspected adrenal disease. Due to the increased use of imaging for diagnostic purposes, adrenal incidentalomas have become a growing clinical problem $(1,2)$. In clinical practice, it is essential to separate the incidentalomas that need intervention, i.e. hormone secreting and malignant tumours, from the majority of incidentalomas that are benign, non-hyperfunctioning lesions that can be left untreated. Adrenocortical cancer has been reported in 1-9\% and hormonal hypersecretion in $2-20 \%$ of patients with adrenal incidentalomas (3-9). So far there are only a limited number of large longitudinal studies on the risk of developing malignancies and hormonal hypersecretion in patients with adrenal incidentaloma $(3,7,8,10-13)$, and there are still no established recommendations for follow-up.

In Sweden, a standardized diagnostic programme was instituted in 1996 by the Swedish Research Council Study Group of Endocrine Abdominal Tumours. The purpose of the programme was to establish patients eligible for surgery by using screening tests that were possible to perform without referring to a specialized unit. In this multicentre study, 381 cases with adrenal incidentaloma were included. After an initial work up, 85 patients had primary surgery 
due to high risk of adrenal malignancy or significant endocrine hyperfunction (9). Of those, 5\% had a benign hypersecreting tumour and nearly $4 \%$ had a malignant tumour. A follow-up of 27-30 months in non-operated patients was recommended to investigate the risk of developing malignant adrenal tumours and clinically overt hypersecreting tumours. In the present study, we report follow-up data in 229 of the initially included 381 patients with adrenal incidentalomas.

\section{Subjects and methods}

New cases of accidentally discovered adrenal masses were registered in Sweden between January 1996 and July 2001. All Swedish hospitals were invited to participate in the study and 381 reported cases from 33 different hospitals were investigated according to a standardized diagnostic programme. Details of the diagnostic programme and characteristics of the included incidentaloma patients have been described in a previous report (9). Recommendations for operation at discovery of the incidentaloma were either an incidentaloma size of more than $3-4 \mathrm{~cm}$ or suspicion of hypersecretion. Eighty-five out of the included 381 patients were initially adrenalectomized (9).

Patients not primarily operated at discovery of the incidentaloma were enrolled in this follow-up study. Out of the initially included 381 patients, 229 patients (98 males and 131 females) were followed with computed tomography (CT). Fifteen of these patients had an incidentaloma size of $5 \mathrm{~cm}$ or more. The reasons for not primarily operating on these patients were: severe concomitant disease $(n=6)$, fine needle biopsy showing benign lesion $(n=4)$, suspicion of haemorrhage with size decrease at follow-up $(n=2)$, followup with CT was chosen instead and operation was performed during the follow-up period $(n=3)$. Twenty-seven different hospitals contributed with follow-up results, which were registered until December 2004. The reasons for lack of follow-up data in the remaining 152 patients are shown in Table 1. According to the follow-up protocol, reinvestigation with

Table 1 The reasons for lack of follow-up data in 152 of the initially included 381 patients.

\begin{tabular}{lc}
\hline & Number \\
\hline Adrenalectomized at discovery & $90^{\mathrm{a}}$ \\
Missing data or declined follow-up & 54 \\
Generalized malignancy & $5^{\mathrm{b}}$ \\
Deceased & $3^{\mathrm{c}}$
\end{tabular}

${ }^{\text {a }}$ After publication of the initial results (9) it has been reported that an additional five patients of the included 381 patients were adrenalectomized at discovery of the incidentaloma; non-hyperfunctioning adrenal adenoma $(n=4)$, non-hyperfunctioning adrenal hyperplasia $(n=1)$.

${ }^{\mathrm{b}}$ Bronchial carcinoma $(n=2)$, colon cancer $(n=2)$, renal cancer $(n=1)$.

${ }^{c}$ Causes of death: malignant melanoma $(n=1)$, cancer of urinary collecting system $(n=1)$, unknown $(n=1)$.
CT scans was recommended at least three times after diagnosis (after 3-6 months, 15-18 months and 27-30 months). All patients with incidentalomas were reinvestigated with CT scans at the same hospital as the initial investigation. In every single patient, the same investigator made size estimates on both the new scan and the previously performed scans. An increase or decrease of incidentaloma size was registered in the protocol. The 229 patients were followed for a median time of 25 months (range 3-108 months). The reasons for not completing all of the recommended CT scans in the follow-up protocol varied: reduction in incidentaloma size $(n=6)$, generalized malignancy $(n=4)$, otherwise severe concomitant disease $(n=7)$, deceased $(n=4)$, patient decline $(n=9)$, operation during follow-up $(n=7)$, missing data or patients not identified $(n=52)$.

Moreover, a hormonal screening evaluation, with 24-h urinary measurements of cortisol, epinephrine, norepinephrine and aldosterone, was recommended at least at 27-30 months after discovery of the incidentaloma. If 24-h urinary aldosterone was elevated, measurement of plasma renin or plasma renin activity was also recommended. Results of the endocrine evaluation were available in 151 of the 229 followed patients.

Adrenalectomy was recommended when there was an increase in incidentaloma size or when a suspicion of Cushing's syndrome, phaeochromocytoma or primary hyperaldosteronism occurred.

Data are presented as median and range. The study was approved by the Ethics Committee.

\section{Results}

The median age of the 229 patients (57\% females) included in the follow-up study was 64 years (range 28-84 years) and the median size of the adrenal incidentalomas when discovered was $2.5 \mathrm{~cm}$ (range $1-8 \mathrm{~cm}$ ) (Fig. 1). The corresponding variables for the 62 not

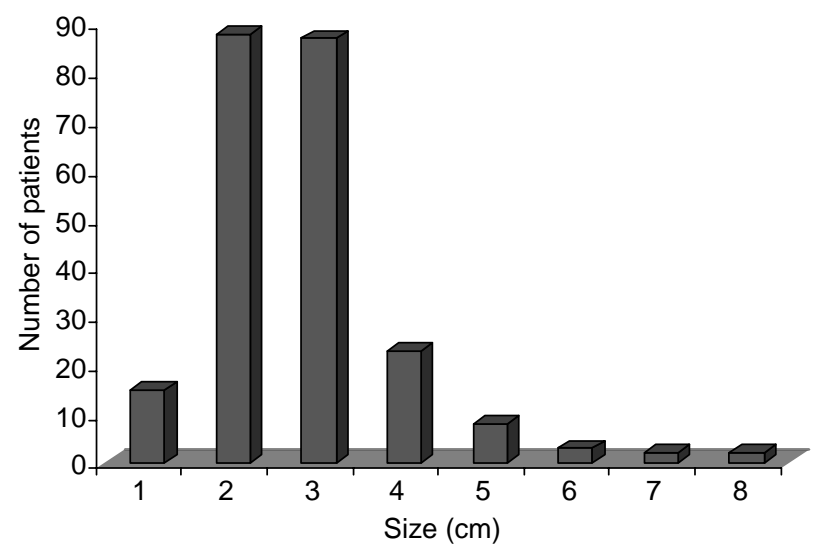

Figure 1 Size of the 229 adrenal incidentalomas included in the follow-up study. 
primarily adrenalectomized patients, lacking any followup data (Table 1), were $61 \%$ females, median age 66.5 years (range 32-84 years) and median size of the incidentalomas $2.5 \mathrm{~cm}$ (range 1.5-6.0 cm). Eighteen of the 229 patients had bilateral incidentalomas.

During the follow-up period, an increase in incidentaloma size of $\geq 0.5 \mathrm{~cm}$ was reported in 17 of the 229 patients $(7.4 \%)$ and an increase of $\geq 1.0 \mathrm{~cm}$ was reported in 12 patients $(5.2 \%)$. The median size at discovery of the 17 incidentalomas with tumour growth was $2.5 \mathrm{~cm}$ (range $1.0-5.5 \mathrm{~cm}$ ) and the median age of the patients was 63 years (range 38-83 years). Eleven of these patients underwent adrenalectomy (Table 2). The reason for operation was tumour growth in seven cases (nos.1-7). Four of these patients were diagnosed with an adrenal adenoma, two with adrenal hyperplasia and one with a myelolipoma. Four of the patients that underwent adrenalectomy were operated mainly due to a rise in hormone secretion (nos. 8-11). Two patients were diagnosed with Cushing's syndrome at 10 and 36 months after the initial diagnosis and both were found to have an adrenal adenoma. Two patients developed a rise in 24-h urinary catecholamines; one of them was diagnosed with a phaeochromocytoma found after 108 months of observation and one was diagnosed with a myelolipoma. No malignant adrenal tumour was diagnosed among the patients operated during follow-up.

Six patients had an increase in incidentaloma size (median $1.1 \mathrm{~cm}$; range $0.5-2.0 \mathrm{~cm}$ ), but were not operated. The size increase was observed 8-28 months (median 17 months) after the incidentalomas were discovered. The reasons for not operating on these patients were severe vascular disease $(n=1)$, generalized pulmonary malignancy $(n=1)$, high age $(n=3)$, and unknown $(n=1)$.

In 12 patients $(5.2 \%)$ there was a reduction in incidentaloma size. In six of these cases there was a total regression of the incidentaloma and these adrenal masses were regarded as haemorrhages. In one case, a size decrease was seen after aspiration of cyst fluid and in the remaining five patients, the reasons for a decrease in size were unknown. In 200 patients $(87.4 \%)$, the incidentaloma size was not altered during the follow-up period.

Four patients died during the follow-up period due to cardiac disease $(n=3)$ or renal failure $(n=1)$. None of these patients had endocrine hyperfunction. Moreover, there was no primary adrenal malignancy among the four patients with reduced follow-up time due to generalized malignancy: bronchial carcinoma $(n=1)$, angiosarcoma $(n=1)$, cervix cancer $(n=1)$, and primary liver cancer $(n=1)$.

\section{Discussion}

Recommendations for follow-up of patients with adrenal incidentalomas are designed to detect malignant and hypersecreting tumours. However, the management of incidentalomas is complicated by the lack of effective diagnostic procedures to distinguish between different aetiologies. There is still controversy regarding the optimal follow-up strategy, as data are based on a limited number of studies, often including only a small group of patients with variable follow-up times (14). Moreover, so far there have only been a few investigations with prespecified follow-up protocols (15-17). In the present study, we evaluated the risk of developing primary adrenal malignancy and endocrine morbidity in 229 patients with adrenal incidentalomas during a median follow-up of 25 months. Limitations of this multicentre study were, however, that endocrine and radiological investigations were not centralized, and due to a lack of reporting clinical data at diagnosis and at time of follow-up, a full phenotyping of patients

Table 2 Characteristics of 11 incidentaloma patients adrenalectomized during follow-up.

\begin{tabular}{|c|c|c|c|c|c|c|c|}
\hline No. & Sex & $\begin{array}{c}\text { Age } \\
\text { (years) }\end{array}$ & $\begin{array}{l}\text { Incidentaloma size } \\
\text { at discovery }{ }^{a}(\mathrm{~cm})\end{array}$ & $\begin{array}{l}\text { Incidentaloma size } \\
\text { at operation }^{\mathrm{b}}(\mathrm{cm})\end{array}$ & $\begin{array}{l}\text { Time between discovery } \\
\text { and operation (months) }\end{array}$ & $\begin{array}{l}\text { Hormonal } \\
\text { evaluation }\end{array}$ & $\begin{array}{l}\text { Histological } \\
\text { diagnosis }\end{array}$ \\
\hline 1 & $\mathrm{M}$ & 38 & 2.5 & 4.0 & 26 & Normal & Myelolipoma \\
\hline 2 & $\mathrm{~F}$ & 46 & 3.0 & 6.5 & 29 & Normal & $\begin{array}{l}\text { Unilateral } \\
\text { macronodular } \\
\text { hyperplasia }\end{array}$ \\
\hline 3 & $\mathrm{~F}$ & 49 & 2.0 & 4.0 & 60 & Normal & Adenoma \\
\hline 5 & $\mathrm{~F}$ & 63 & 2.5 & 3.0 & 13 & Normal & Adenoma \\
\hline 6 & $\mathrm{~F}$ & 64 & 1.7 & 4.5 & 22 & Normal & Hyperplasia \\
\hline 7 & $\mathrm{~F}$ & 67 & 4.5 & 5.5 & 9 & Normal & Adenoma \\
\hline 8 & $\mathrm{~F}$ & 51 & 1.0 & 1.5 & 10 & U-cortisol $\uparrow$ & Adenoma \\
\hline 9 & $\mathrm{~F}$ & 56 & 1.5 & 2.0 & 108 & $\begin{array}{l}\text { U-norepinephrine } \uparrow \\
\text { U-epinephrine } \uparrow\end{array}$ & $\begin{array}{l}\text { Phaeochromo- } \\
\text { cytoma }\end{array}$ \\
\hline 10 & $\mathrm{~F}$ & 60 & 5.0 & 7.0 & 21 & U-norepinephrine $\uparrow$ & Myelolipoma \\
\hline 11 & $\mathrm{~F}$ & 61 & 3.0 & 5.7 & 36 & U-cortisol $\uparrow$ & Adenoma \\
\hline
\end{tabular}

${ }^{\mathrm{a}}$ Maximal diameter measured at a computed tomography examination; ${ }^{\mathrm{b}}$ Maximal diameter measured after surgical excision.

U, urinary. 
with adrenal incidentalomas was not possible in this study. Furthermore, only $79 \%$ of the patients not primarily adrenalectomized were followed with CT scans. The characteristics (sex, age and incidentaloma size) of the 62 patients without available follow-up data and the 229 followed patients were, however, similar, which suggests that the conclusions of this data are valid in spite of this group of subjects lost to follow-up.

In agreement with previous investigations (18), we found that most adrenal incidentalomas remained stable in size $(87.4 \%)$. In a previous review of 18 published series including 873 patients, mass enlargement greater than $1 \mathrm{~cm}$ was found in $0-26.4 \%$ of patients (14). In the present study, there was an increase in incidentaloma size of $0.5 \mathrm{~cm}$ or more in $7.4 \%$ and of $1 \mathrm{~cm}$ or more in $5.2 \%$ of patients. The higher percentage of tumour growth in some previous reports might be explained by a longer follow-up period (13, 16, 17), as the cumulative risk of mass enlargement seems to increase with time (17). The size of the adrenal incidentaloma was described as the maximal diameter of the lesion. It is, however, important to emphasize that the increase in the diameter underestimates the increase in tumour volume.

In the present investigation, 11 of the 17 patients with incidentaloma growth were adrenalectomized. However, none of them was diagnosed with an adrenal carcinoma. Increase in size has been considered suggestive of malignancy (19). Our results confirm, however, previous findings from longitudinal studies showing that the risk for developing adrenal carcinoma is very low, despite an increase in incidentaloma size $(3,5,7$, $8,10,12,13,15,16,20)$. Although we found no cases of primary adrenal malignancy during followup, altogether seven of the patients lacking follow-up data, as well as four of the 229 followed patients (one increased in size) were diagnosed with generalized malignant disease. None of these patients underwent adrenalectomy, but it is possible that in some of these cases the adrenal mass represented a metastasis.

In the presently used follow-up programme, the estimate from the CT investigation was size. After the introduction of this programme in 1996, other diagnostic parameters have been shown to have a potential to differentiate between benign and malignant lesions, such as homogeneity, distinctness of margins and CT attenuation. For example, it has been stated that a homogenous mass with a smooth border and a low attenuation value is highly suggestive of a benign adenoma (21). Inclusion of such diagnostic imaging tools in a future follow-up programme could therefore play an important role in ruling out malignancies at an early stage, thus shortening the follow-up time in selected cases.

In the present study, a hypersecreting tumour was diagnosed in only $2 \%$ of the hormonally investigated patients: two patients with cortisol-secreting adenomas and clinical Cushing's syndrome and one patient with phaeochromocytoma. This shows that overt hormonal oversecretion is rare during a two-year follow-up of adrenal incidentalomas. However, the diagnosis of subclinical cortisol hypersecretion might have been underestimated in this study, because our diagnostic follow-up programme, which was instituted in 1996, only included 24-h urinary sampling of cortisol for screening of hypercortisolism. In recent studies, with a more detailed evaluation of the hypothalamus-pituitary-adrenal axis, patients with mild autonomous cortisol production without specific clinical signs of cortisol excess have been identified. This subclinical Cushing's syndrome, not possible to detect in our study, has been shown to develop in up to $20 \%$ of patients (21).

Previously, it has been reported that the risk for tumour hyperfunction seems to plateau after three to four years (17). In the present study, the two patients with Cushing's syndrome were diagnosed within this time period, whereas in the patient diagnosed with phaeochromocytoma, a rise in urinary catecholamines was found after 108 months of observation. In the present study, none of the patients developed primary hyperaldosteronism, which is in accordance with previous results, revealing no cases of primary hyperaldosteronism during follow-up in patients with adrenal incidentaloma (14).

In the present investigation one patient with a myelolipoma was operated upon under the suspicion of phaeochromocytoma, due to a rise in 24-h urinary norepinephrine. Recent investigations have shown that plasma free metanephrines can be measured with high diagnostic accuracy and it has therefore been suggested that this test may replace or complement the measurement of urine catecholamines for the diagnosis of phaeochromocytoma in patients with incidentaloma $(18,21)$. Moreover, the plasma aldosterone/plasma renin ratio is a sensitive and specific tool to evaluate potential cases of primary hyperaldosteronism (22) and it has therefore been proposed that this test also should be included in a future follow-up programme for patients with adrenal incidentaloma (18).

In conclusion, in this Swedish prospective short-term follow-up study no cases of primary adrenal malignancy were diagnosed. Only $2 \%$ of the hormonally investigated patients were diagnosed with endocrine hyperfunction during follow-up. More long-term investigations of patients with adrenal incidentaloma are, however, warranted in order to study the natural course of adrenal incidentalomas. In these future investigations it is desirable that measurement of mass density is included, and that biochemical tests with higher diagnostic accuracy for diagnosis of endocrine hyperfunction should be used.

\section{Acknowledgements}

The authors wish to thank Professor Sigbritt Werner, Dept. of Endocrinology, Metabolism and Diabetology, Karolinska University Hospital, Huddinge, Sweden, for 
invaluable comments on the establishment of the programme. The authors are also grateful to the Swedish Research Council for the long-term support of the Study Group of Encocrine Abdominal Tumours and to all clinicians reporting the subjects. (1) Borås $(n=5$; L Norrman, H Widell); (2) Danderyd ( $n=1$; L Eggimann); (3) Eksjö ( $n=1$; J Järhult); (4) Eskiltuna ( $n=18$; P Gillberg, L Steen); (5) Helsingborg $(n=4$; H Mårtensson); (6) Karolinska University Hospital, Huddinge ( $n=15$; H Lundin, E Moberg, M Rydén, K Steiner, H Wahrenberg, S Werner); (7) Karolinska University Hospital, Solna $(n=42$; M Alvarsson, J Calissendorff, M Degerblad, B Freyschuss, G Jörneskog, M Thorén, H Wagner); (8) Jönköping ( $n=3$; J Järhult); (9) Kungsbacka ( $n=2$; N-B Rangvin, G Stenström); (10) Lidköping ( $n=1$; K Fredricson); (11) Linköping ( $n=8$; LG Arnesson, J Blomgren, M Dovrén-Rustcheff, B Ekman, G Toss, P Öhman); (12) Ljungby $(n=1 ; \mathrm{H}$ Larsson); (13) Luleå/Boden $(n=10 ; \mathrm{M}$ Eliasson, E Enbuska, M Gjörup, S Tollqvist); (14) Lund ( $n=45$; P Hedner, S Valdemarsson, A Wihl, B Bülow); (15) Malmö ( $n=24$; M Bramnert, M Ekelund, L Fredstorp, B Hallengren, L Hulthén, $\mathrm{H}$ Larsson, $\mathrm{P}$ Lind, $\mathrm{P}$ Manhem, M Ridderstråle, A Scott, T Tuomi); (16) Motala ( $n=1$; M Strand); (17) Norrköping $(n=1$; A-M Ottosson); (18) NÄL ( $n=1$; S Ander); (19) Göteborg ( $n=18$; H Filipsson, $\mathrm{S}$ Jansson, $\mathrm{V}$ Johanson, L Kölby, B Wängberg); (20) St Göran's Hospital, Stockholm ( $n=1$; I Linder); (21) Sundsvall ( $n=4$; K Andersson, L Engström); (22) Umeå $(n=5$; E Hägg); (23) Uppsala ( $n=12$; C Juhlin); (24) Visby ( $n=2$; E Forbes); (25) Värnamo $(n=2$; K Wahlin); (26) Västerås $(n=1 ; \AA$ Tenerz); (27) Ystad $(n=1$; G Tallroth).

\section{References}

1 Gross MD \& Shapiro B. Clinical review 50. Clinically silent adrenal masses. Journal of Clinical Endocrinology and Metabolism 199377 $885-888$.

2 Chidiac RM \& Aron DC. Incidentalomas. A disease of modern technology. Endocrinology and Metabolism Clinics of North America $199726233-253$.

3 Herrera MF, Grant CS, van Heerden JA, Sheedy PF \& Ilstrup DM. Incidentally discovered adrenal tumors: an institutional perspective. Surgery $19911101014-1021$.

4 Osella G, Terzolo M, Borretta G, Magro G, Ali A, Piovesan A, Paccotti P \& Angeli A. Endocrine evaluation of incidentally discovered adrenal masses (incidentalomas). Journal of Clinical Endocrinology and Metabolism 199479 1532-1539.

5 Bastounis EA, Karayiannakis AJ, Anapliotou ML, Nakopoulou L, Makri GG \& Papalambros EL. Incidentalomas of the adrenal gland: diagnostic and therapeutic implications. American Surgeon 199763 356-360.

6 Favia G, Lumachi F, Basso S \& D'Amico DF. Management of incidentally discovered adrenal masses and risk of malignancy. Surgery $2000128918-924$.
7 Mantero F, Terzolo M, Arnaldi G, Osella G, Masini AM, Ali A, Giovagnetti M, Opocher G \& Angeli A. A survey on adrenal incidentaloma in Italy. Journal of Clinical Endocrinology and Metabolism $200085637-644$.

8 Barzon L, Fallo F, Sonino N \& Boscaro M. Development of overt Cushing's syndrome in patients with adrenal incidentalomas. European Journal of Endocrinology 2002146 61-66.

9 Bülow B \& Ahrén B on behalf of the Swedish Research Council Study Group of Endocrine Abdominal Tumours, Adrenal incidentaloma - experience of a standardized diagnostic programme in the Swedish prospective study. Journal of Internal Medicine 2002 $252239-246$.

10 Barry MK, van Heerden JA, Farley DR, Grant CS, Thompson GB \& Ilstrup DM. Can adrenal incidentalomas be safely observed? World Journal of Surgery 199822 599-604.

11 Terzolo M, Osella G, Ali A, Borretta G, Cesario F, Paccotti P \& Angeli A. Subclinical Cushing's syndrome in adrenal incidentaloma. Clinical Endocrinology 1998 48 89-97.

12 Grossrubatscher E, Vignati F, Possa M \& Lohi P. The natural history of incidentally discovered adrenocortical adenomas: a retrospective evaluation. Journal of Endocrinological Investigation $200124846-855$.

13 Libè R, Dall'Asta C, Barbetta L, Baccarelli A, Beck-Peccoz P \& Ambrosi B. Long-term follow-up study of patients with adrenal incidentalomas. European Journal of Endocrinology 2002147 489-494.

14 Barzon L, Sonino N, Fallo F, Palù G \& Boscaro M. Prevalence and natural history of adrenal incidentalomas. European Journal of Endocrinology 2003149 273-285.

15 Jockenhovel F, Kuck W, Hauffa B, Reinhardt W, Benker G, Lederbogen S, Olbricht T \& Reinwein D. Conservative and surgical management of incidentally discovered adrenal tumors (incidentalomas). Journal of Endocrinological Investigation $1992 \mathbf{1 5}$ $331-337$.

16 Sirén J, Tervahartiala P, Sivula A \& Haapiainen R. Natural course of adrenal incidentalomas: seven-year follow-up study. World Journal of Surgery 200024 579-582.

17 Barzon L, Scaroni C, Sonino N, Fallo F, Paoletta A \& Boscaro M. Risk factors and long-term follow-up of adrenal incidentalomas. Journal of Clinical Endocrinology and Metabolism $1999 \mathbf{8 4}$ $520-526$.

18 Mansmann G, Lau J, Balk E, Rothberg M, Miyachi Y \& Bornstein SR. The clinically inapparent adrenal mass: update in diagnosis and management. Endocrine Review 200425 309-340.

19 Copeland PM. The incidentally discovered adrenal mass. Annals of Internal Medicine 198398 940-945.

20 Rossi R, Tauchmanova L, Luciano A, Di Martino M, Battista C, Del Viscovo L, Nuzzo V \& Lombardi G. Subclinical Cushing's syndrome in patients with adrenal incidentaloma: clinical and biochemical features. Journal of Clinical Endocrinology and Metabolism 200085 1440-1448.

21 Grumbach MM, Biller BM, Braunstein GD, Campbell KK, Carney JA, Godley PA, Harris EL, Lee JK, Oertel YC, Posner MC, Schlechte JA \& Wieand HS. Management of the clinically inapparent adrenal mass ("incidentaloma") [NIH Conference]. Annals of Internal Medicine 2003138 424-429.

22 Young WF Jr, Hogan MJ, Klee GG, Grant CS \& van Heerden JA. Primary aldosteronism: diagnosis and treatment. Mayo Clinic Proceedings 199065 96-110.

Received 18 October 2005

Accepted 21 December 2005 